\title{
Bassalygo-Dobrushin uniqueness for continuous spin systems on irregular graphs
}

\author{
D.Kȩpa*Yu.Kozitsky ${ }^{\dagger}$ \\ Instytut Matematyki, Uniwersytet Marii Curie-Skłodowskiej, 20-031 Lublin, Poland
}

Received January 31, 2008

An extension of the Bassalygo-Dobrushin technique of proving uniqueness of Gibbs fields on irregular graphs, developed in [Theory of Probab. Appl. 31, 572-589 (1986)], to the case of continuous spins has been presented.

Key words: Gibbs random field, Gibbs specification, Polish space, sparse graph

PACS: $05.20 . \mathrm{Gg}, 05.30 . \mathrm{Ch}$

\section{Introduction and setup}

The theory of Gibbs random fields indexed by countable sets (e.g., Gibbs states of lattice models) is now well elaborated, see [1]. At the same time, only a few papers deal with the field having index sets presented by irregular graphs. Among them there is a paper by L. A. Bassalygo and R. L. Dobrushin, [2]. It presents a technique of proving uniqueness if the single-site state spaces (single-spin spaces) are finite. Our aim is to develop a similar technique, which covers the case where the single-site state spaces are general metric spaces. In this case, with slight abuse of terminology we say that the spins are continuous, which is reflected in the title above. The basic assumption, however, is that the interaction between the spins is bounded. As possible applications of our theory we mention the theory of Euclidean Gibbs states of the following quantum models. In each of them, quantum particles are located at sites (one particle per site), which form an irregular structure. The single-particle Hamiltonians have discrete spectra and the interparticle interaction is pair-wise and bounded. As a quantum particle, one can take: (a) a free particle moving in a compact Riemannian manifold (e.g. quantum rotator), see [3,4]; (b) a free particle moving in a compact subset of $\mathbb{R}^{d} ;(\mathrm{c})$ a quantum anharmonic oscillator, see e.g. [5].

Let (L, E) be a graph with (infinite) countable sets of vertices, L, and edges, E. We also suppose that the graph is simple, i.e., it has no loops, isolated vertices, and multiple edges. Two vertices $\ell, \ell^{\prime}$ are called adjacent if there exists an edge $\left\langle\ell, \ell^{\prime}\right\rangle$. The number $n_{\ell}$ of the vertices adjacent to $\ell$ is called degree. For each $\ell \in \mathrm{L}$, let $X_{\ell}$ be a complete separable metric space (Polish space), $\mathcal{B}\left(X_{\ell}\right)$ be the corresponding Borel $\sigma$-field, and $\chi_{\ell}$ be a finite Borel measure on $\left(X_{\ell}, \mathcal{B}\left(X_{\ell}\right)\right)$. For an edge $\left\langle\ell, \ell^{\prime}\right\rangle$, let a bounded symmetric continuous function (potential) $V_{\ell \ell^{\prime}}: X_{\ell} \times X_{\ell^{\prime}} \rightarrow \mathbb{R}$ be given. Under certain conditions, these objects define a Gibbs random field on the product space $X=\prod_{\ell \in \mathrm{L}} X_{\ell}$. If all $V_{\ell \ell^{\prime}}$ equal zero, there exists only one Gibbs field. Thus, one can expect the same uniqueness if the potentials are sufficiently small, which certainly depends on the underlying graph. If the latter is regular (each vertex has the same degree), the proof of the uniqueness by small potentials is quite standard. The case where $n_{\ell}$ 's are different but globally bounded (there exists $\hat{n} \in \mathbb{N}$, such that $n_{\ell} \leqslant \hat{n}$ for all $\ell \in \mathrm{L}$ ) can be handled similarly. The situation changes substantially if $\sup _{\ell \in \mathrm{L}} n_{\ell}=+\infty$. This can be seen from the example considered in Section 4 below, where the graph is so dense that the ferromagnetic Ising model defined on this graph has multiple Gibbs states for arbitrary non-zero interactions. For sparse graphs of a certain kind, which in

\footnotetext{
*E-mail: dkepa@hektor.umcs.lublin.pl

${ }^{\dagger}$ E-mail: jkozi@hektor.umcs.lublin.pl
} 
particular means that the vertices of large degree are at large distances from each other, we prove that the number of Gibbs fields with Polish single-spin spaces is exactly one if the potentials are small enough. The proof is based on an extension and refinement of the technique developed in [2], where all $X_{\ell}$ were finite.

For a finite set $A$, by $|A|$ we denote its cardinality (the number of elements in $A$ ). Let $\mathfrak{L}_{\text {fin }}$ stand for the family of all finite non-void subsets of the vertex set $\mathrm{L}$. A property related with a given $\Lambda \in \mathfrak{L}_{\text {fin }}$ is called local, whereas global properties are going to be related with the whole graph. If we say that something holds for all $\ell$ (resp. for all $e$ ), it holds for all $\ell \in \mathrm{L}$ (resp. all $e \in \mathrm{E}$ ). As usual, for $\Lambda \subset \mathrm{L}$, we write $\Lambda^{c}=\mathrm{L} \backslash \Lambda$. A sequence $\mathcal{D} \subset \mathfrak{L}_{\text {fin }}$ is called cofinal if it is ordered by inclusion and exhausts $\mathrm{L}$. The latter means that any $\Lambda \in \mathfrak{L}_{\text {fin }}$ is contained in a certain $\Delta \in \mathcal{D}$. The limits taken along such a sequence will be denoted by $\lim _{\mathcal{D}}$. For $\Lambda \subset \mathrm{L}$, we set

$$
\begin{aligned}
\mathrm{E}_{\Lambda} & =\left\{\left\langle\ell, \ell^{\prime}\right\rangle \in \mathrm{E} \mid \ell, \ell^{\prime} \in \Lambda\right\}, \\
\partial_{\mathrm{E}} \Lambda & =\left\{\left\langle\ell, \ell^{\prime}\right\rangle \in \mathrm{E} \mid \ell \in \Lambda, \ell^{\prime} \in \Lambda^{c}\right\}, \\
\partial_{\mathrm{L}} \Lambda & =\left\{\ell^{\prime} \in \Lambda^{c} \mid \exists \ell \in \Lambda:\left\langle\ell, \ell^{\prime}\right\rangle \in \mathrm{E}\right\} .
\end{aligned}
$$

The latter sets are called the edge and the vertex boundary of $\Lambda$, respectively. For a one-point $\Lambda=$ $\{\ell\}$, its edge and vertex boundaries are written $\partial_{\mathrm{E}} \ell$ and $\partial_{\mathrm{L}} \ell$ respectively. Clearly, the cardinalities of these both sets coincide, that is, $\left|\partial_{\mathrm{E}} \ell\right|=\left|\partial_{\mathrm{L}} \ell\right|$. We suppose that the graph is locally finite, which means that, for every $\ell$, its degree $n_{\ell} \stackrel{\text { def }}{=}\left|\partial_{\mathrm{E}} \ell\right|$ is finite. Therefrom it follows that

$$
\left|\partial_{\mathrm{L}} \Lambda\right| \leqslant\left|\partial_{\mathrm{E}} \Lambda\right|<\infty
$$

for each $\Lambda \in \mathfrak{L}_{\text {fin }}$. For $\Lambda \subset \mathrm{L}$ and $\ell, \ell^{\prime} \in \Lambda \cup \partial_{\mathrm{L}} \Lambda$, the sequence $\vartheta\left(\ell, \ell^{\prime}\right)=\left\{\ell_{0}, \ell_{1}, \ldots, \ell_{n}\right\}$, such that $\ell_{0}=\ell, \ell_{n}=\ell^{\prime}, \ell_{1}, \ldots \ell_{n-1} \in \Lambda$, is called a path in $\Lambda$ if $\left\langle\ell_{k}, \ell_{k+1}\right\rangle \in \mathrm{E}$ for all $k=0,1, \cdots, n-1$. In principle, a path may intersect itself, that is, certain vertices $\ell_{k}$ may appear in $\vartheta\left(\ell, \ell^{\prime}\right)$ more than once. The length of a path, $\left|\vartheta\left(\ell, \ell^{\prime}\right)\right|$, is set to be the number of pairs of consecutive vertices; thus, in the situation above we had $\left|\vartheta\left(\ell, \ell^{\prime}\right)\right|=n$. A path in $\mathrm{L}$ is merely called a path. By $\rho\left(\ell, \ell^{\prime}\right)$ we denote the distance between $\ell$ and $\ell^{\prime}$ - the length of the shortest path connecting these vertices.

Definition 1.1. A path $\vartheta\left(\ell, \ell^{\prime}\right)$ is called a self-avoiding if it has no self-intersections. This means that each $\ell_{i}$ appears in $\vartheta\left(\ell, \ell^{\prime}\right)$ only once. Thus, in a self-avoiding path, only the endpoints $\ell_{0}, \ell_{n}$ can have degree 1.

For a vertex $\ell$ and $N \in \mathbb{N}$, let $\mathcal{L}_{N}(\ell)$ be the set of all self-avoiding paths of length $N$ originating at $\ell$.

Definition 1.2. The graph is called sparse if there exist positive constants $C$ and $\eta$ such that, for every vertex $\ell$, there exists $N_{\ell} \in \mathbb{N}$ such that, for all $N \geqslant N_{\ell}$,

$$
\left|\mathcal{L}_{N}(\ell)\right| \leqslant C \eta^{N}
$$

A particular case of locally bounded graphs was introduced in [2]. For each of them,

$$
\rho\left(\ell, \ell^{\prime}\right) \geqslant \phi\left(\min \left\{n_{\ell}, n_{\ell^{\prime}}\right\}\right)
$$

where $\phi: \mathbb{N} \rightarrow[1,+\infty)$ is an increasing function, specific for the graph, such that

$$
\sum_{k=1}^{+\infty} \frac{k}{\phi\left(2^{k}\right)}<\infty
$$

By (1.3), two vertices of large degrees repel each other. Below, see Lemma 3.2, we prove that such graphs are sparse in the sense of Definition 1.2.

For a topological space $Y$, by $C_{\mathrm{b}}(Y)$ we denote the set of all bounded continuous functions $f: Y \rightarrow \mathbb{R}$, and by $\mathcal{B}(Y)$ - the corresponding Borel $\sigma$-field. By saying that $\mu$ is a measure on $Y$, we 
mean that $\mu$ is a measure on the measurable space $(Y, \mathcal{B}(Y))$. The set of all probability measures on $Y$ is denoted by $\mathcal{P}(Y)$. For a measurable function $f: Y \rightarrow \mathbb{R}$, we write

$$
\mu(f)=\int_{Y} f \mathrm{~d} \mu .
$$

Let $X_{\ell}, \ell \in \mathrm{L}$, be the Polish spaces mentioned above. For $\Delta \subseteq \mathrm{L}$, the Cartesian product

$$
X_{\Delta}=\prod_{\ell \in \Delta} X_{\ell}
$$

is equipped with the product topology, so that $\left(X_{\Delta}, \mathcal{B}\left(X_{\Delta}\right)\right)$ becomes a standard Borel space. This means that there exists a Polish space $Y$ and a measurable isomorphism $\varphi: X_{\Delta} \rightarrow Y$. For more details we refer the reader to section 4.A, page 73 of [1]. The elements of $X_{\Delta}$ are denoted by $x_{\Delta}$; we write $X=X_{\mathrm{L}}$ and $x=x_{\mathrm{L}}$. For $\Lambda \subset \Delta$, the just a position $x_{\Delta}=x_{\Lambda} \times x_{\Delta \backslash \Lambda}$ defines an embedding $X_{\Lambda} \hookrightarrow X_{\Delta}$ by identifying $x_{\Lambda}$ with $x_{\Lambda} \times x_{\Delta \backslash \Lambda}^{0}$, where $x^{0}$ is a certain fixed element of $X$. In view of this embedding, one has $\mathcal{B}\left(X_{\Lambda}\right) \subset \mathcal{B}\left(X_{\Delta}\right)$; thus, one can consider

$$
\mathcal{B}^{\text {loc }}=\bigcup_{\Lambda \in \mathfrak{L}_{\text {fin }}} \mathcal{B}\left(X_{\Lambda}\right)
$$

which is called the $\sigma$-field of local events. The tail $\sigma$-field is defined to be

$$
\mathcal{B}^{\text {tail }}=\bigcap_{\Lambda \in \mathfrak{L}_{\text {fin }}} \mathcal{B}\left(X_{\Lambda^{c}}\right)
$$

Suppose now that we have given finite Borel measures $\chi_{\ell}, \ell \in \mathrm{L}$, and symmetric bounded continuous functions $V_{\ell \ell^{\prime}}: X_{\ell} \times X_{\ell^{\prime}} \rightarrow \mathbb{R},\left\langle\ell, \ell^{\prime}\right\rangle \in \mathrm{E}$. As usual, we write

$$
\left\|V_{\ell \ell^{\prime}}\right\|=\sup _{x_{\ell} \in X_{\ell}, x_{\ell^{\prime}} \in X_{\ell^{\prime}}}\left|V_{\ell \ell^{\prime}}\left(x_{\ell}, x_{\ell^{\prime}}\right)\right| .
$$

For $\Lambda \in \mathfrak{L}_{\text {fin }}$, we set

$$
V_{\Lambda}\left(x_{\Lambda} \mid y\right)=\sum_{\left\langle\ell, \ell^{\prime}\right\rangle \in \mathrm{E}_{\Lambda}} V_{\ell \ell^{\prime}}\left(x_{\ell}, x_{\ell^{\prime}}\right)+\sum_{\left\langle\ell, \ell^{\prime}\right\rangle \in \partial_{\mathrm{E}} \Lambda} V_{\ell \ell^{\prime}}\left(x_{\ell}, y_{\ell^{\prime}}\right), \quad y \in X
$$

Clearly, $V_{\Lambda}(\cdot \mid y) \in C_{\mathrm{b}}\left(X_{\Lambda}\right)$ for every $y \in X$. Furthermore, for $\Lambda \in \mathfrak{L}_{\text {fin }}, y \in X$, and $B \in \mathcal{B}(X)$, we set

$$
\pi_{\Lambda}(B \mid y)=\frac{1}{Z_{\Lambda}(y)} \int_{X_{\Lambda}} \mathbb{I}_{B}\left(x_{\Lambda} \times y_{\Lambda^{c}}\right) \exp \left[V_{\Lambda}\left(x_{\Lambda} \mid y\right)\right] \chi_{\Lambda}\left(\mathrm{d} x_{\Lambda}\right),
$$

where $\mathbb{I}_{B}$ is the indicator of $B$,

$$
\chi_{\Lambda}=\bigotimes_{\ell \in \Lambda} \chi_{\ell}
$$

and

$$
Z_{\Lambda}(y)=\int_{X_{\Lambda}} \exp \left[V_{\Lambda}\left(x_{\Lambda} \mid y\right)\right] \chi_{\Lambda}\left(\mathrm{d} x_{\Lambda}\right)
$$

Clearly, each such $\pi_{\Lambda}$ is a probability kernel on $(X, \mathcal{B}(X))$. This means that $\pi_{\Lambda}(\cdot \mid y) \in \mathcal{P}(X)$ for any $y \in X$, and $\pi_{\Lambda}(B \mid \cdot)$ is measurable for any $B \in \mathcal{B}(X)$. The family $\left\{\pi_{\Lambda}\right\}_{\Lambda \in \mathfrak{L}_{\text {fin }}}$ defines a Gibbs random field on the graph in the following sense. By construction,

$$
\pi_{\Delta}(\cdot \mid y)=\int_{X} \pi_{\Lambda}(\cdot \mid x) \pi_{\Delta}(\mathrm{d} x \mid y)
$$

which holds for any $\Lambda \subset \Delta$. 
Definition 1.3. A probability measure $\mu$ on $X$ is called a Gibbs random field if for every $\Lambda \in \mathfrak{L}_{\text {fin }}$,

$$
\mu(\cdot)=\int_{X} \pi_{\Lambda}(\cdot \mid x) \mu(\mathrm{d} x) .
$$

The set of all such Gibbs random fields will be denoted by $\mathcal{G}$. If necessary, we write $\mathcal{G}(V)$ to indicate the dependence on the choice of $V=\left(V_{\ell \ell^{\prime}}\right)_{\left\langle\ell, \ell^{\prime}\right\rangle \in \mathrm{E}}$. The following property, the proof of which is quite standard, gives an important information about $\mathcal{G}$.

Proposition 1.4 (Feller Property). For every $\Lambda \in \mathfrak{L}_{\text {fin }}$ and $f \in C_{\mathrm{b}}(X)$, it follows that $\pi_{\Lambda}(f \mid \cdot) \in$ $C_{\mathrm{b}}(X)$.

Given $y \in X$, let $\mathcal{P}_{y}$ be the family of the accumulation points of $\left\{\pi_{\Lambda}(\cdot \mid y)\right\}_{\Lambda \in \mathfrak{L}_{\mathrm{fin}}}$ endowed with the usual weak topology defined by $C_{\mathrm{b}}(X)$.

Corollary 1.5. For any $y \in X$, it follows that $\mathcal{P}_{y} \subset \mathcal{G}$.

Proof. As $C_{\mathrm{b}}(X)$ is a measure defining class, the inclusion in question can be obtained by showing that, for every $\mu \in \mathcal{P}_{y}$ and $\Lambda \in \mathfrak{L}_{\text {fin }}$,

$$
\mu(f)=\int_{X} \pi_{\Lambda}(f \mid x) \mu(\mathrm{d} x) .
$$

By supposition, there exists a cofinal sequence $\mathcal{D}$ such that, for all $f \in C_{\mathrm{b}}(X), \mu(f)=\lim _{\mathcal{D}} \pi_{\Delta}(f \mid y)$. Let $\Lambda$ be as in (1.14). Then one finds $\Delta_{0} \in \mathcal{D}$ such that $\Lambda \subset \Delta$ for all $\Delta \in \mathcal{D}$ starting from this $\Delta_{0}$. For such $\Delta$, one has

$$
\pi_{\Delta}(f \mid y)=\int_{X} \pi_{\Lambda}(f \mid x) \pi_{\Delta}(\mathrm{d} x \mid y)
$$

Passing here to the limit along $\mathcal{D}$ and taking into account that $\pi_{\Lambda}(f \mid \cdot) \in C_{\mathrm{b}}(X)$, one gets (1.14).

This statement allows for the following generalization. By Definition 1.3, a convex combination of Gibbs fields is again a Gibbs field. Let $\mathcal{G}^{\text {ex }}$ be the set of all extreme elements of $\mathcal{G}$, i.e., those which cannot be presented as nontrivial convex combinations of other elements of $\mathcal{G}$. If $\mathcal{G} \neq \emptyset$, then $\mathcal{G}^{\text {ex }} \neq \emptyset$ and each Gibbs field can uniquely be represented as a convex combination of the elements of $\mathcal{G}^{\text {ex }}$, see Theorem 7.26, page 133 in [1]. In particular, $|\mathcal{G}|=1$ if $\left|\mathcal{G}^{\text {ex }}\right|=1$. Given $\mu \in \mathcal{G}$ is extreme if and only if it is trivial on the tail $\sigma$-field (1.7), see Theorem 7.7, page 118 in [1]. This means that $\mu(A)=1$ or $\mu(A)=0$ for any $A \in \mathcal{B}^{\text {tail }}$.

Proposition 1.6. For every $\mu \in \mathcal{G}^{\text {ex }}$ and any cofinal sequence $\mathcal{D}$, the sequence $\left\{\pi_{\Delta}(\cdot \mid y)\right\}_{\Delta \in \mathcal{D}}$ converges weakly to $\mu$ for all $y \in A$, where $A \in \mathcal{B}^{\text {tail }}$ may depend on $\mathcal{D}$ and is such that $\mu(A)=1$.

Proof. For any $f \in C_{\mathrm{b}}(X)$, one has $\lim _{\mathcal{D}} \pi_{\Delta}(f \mid y)=\mu(f)$ for all $y \in A_{f}$, such that $A_{f} \in \mathcal{B}^{\text {tail }}$ and $\mu\left(A_{f}\right)=1$, see claim (a) of Theorem 7.12, page 122 in [1]. But the weak topology is metrizable. Therefore, there exists a countable set $\left\{f_{n}\right\}_{n \in \mathbb{N}} \subset C_{\mathrm{b}}(X)$ such that the fact $\lim _{\mathcal{D}} \pi_{\Delta}\left(f_{n} \mid y\right)=\mu\left(f_{n}\right)$, for all $n \in \mathbb{N}$, yields the convergence in question. Thus, as the set $A$ mentioned above one can take the intersection of all $A_{f_{n}}$.

Proposition 1.7. The set $\mathcal{G}$ is non-void.

Proof. In view of Corollary 1.5, to get the property in question it is enough to show that $\mathcal{P}_{y} \neq \emptyset$ for some $y \in X$. As the spaces $X_{\ell}$ are Polish, for every $\varepsilon_{\ell}>0$, one finds a compact $A_{\ell}\left(\varepsilon_{\ell}\right) \subset X_{\ell}$ such that $\chi_{\ell}\left(X_{\ell} \backslash A_{\ell}\left(\varepsilon_{\ell}\right)<\varepsilon_{\ell} \cdot \chi_{\ell}\left(X_{\ell}\right)\right.$. By Prohorov's theorem, $\mathcal{P}_{y} \neq \emptyset$ if the family $\left\{\pi_{\Delta}(\cdot \mid y)\right\}_{\Delta \in \mathfrak{L}_{\mathrm{fin}}}$ is uniformly tight. Set

$$
v_{\ell}=\sum_{\ell^{\prime} \in \partial_{\llcorner} \ell}\left\|V_{\ell \ell^{\prime}}\right\|
$$

Given $\varepsilon>0$, we pick $\left\{\varepsilon_{\ell}\right\}_{\ell \in \mathrm{L}}$ such that

$$
\varepsilon=\sum_{\ell} \varepsilon_{\ell} e^{2 v_{\ell}}
$$


Thereby, for $\Lambda \in \mathfrak{L}_{\text {fin }}$, we set

$$
C_{\Lambda}^{\varepsilon}=\left\{x \in X \mid \forall \ell \in \Lambda^{c}: \quad x_{\ell} \in A_{\ell}\left(\varepsilon_{\ell}\right)\right\}
$$

Furthermore, we write $C^{\varepsilon}=C_{\emptyset}^{\varepsilon}$ and $C_{\ell}^{\varepsilon}=C_{\{\ell\}}^{\varepsilon}$. Clearly, $C^{\varepsilon}$ is compact. Now let $y \in C_{\Lambda}^{\varepsilon}$ for some $\Lambda \in \mathfrak{L}_{\mathrm{fin}}$. By (1.9), it follows that, for any $\ell$ and $z \in X$,

$\pi_{\{\ell\}}\left(C^{\varepsilon} \mid z\right)=\mathbb{I}_{C_{\ell}^{\varepsilon}}(z)\left[1-\frac{1}{Z_{\ell}(y)} \int_{X_{\ell} \backslash A_{\ell}\left(\varepsilon_{\ell}\right)} \exp \left(\sum_{\ell^{\prime} \in \partial_{\mathrm{L} \ell}} V_{\ell \ell^{\prime}}\left(x_{\ell}, z_{\ell^{\prime}}\right)\right) \chi_{\ell}\left(\mathrm{d} x_{\ell}\right)\right] \mathbb{I}_{C_{\ell}^{\varepsilon}}(z)\left[1-\varepsilon_{\ell} e^{2 v_{\ell}}\right]$.

Integrating both sides of this estimate by (1.12) we get for $\Delta \in \mathfrak{L}_{\text {fin }}$ containing this $\ell$

$$
\pi_{\Delta}\left(C^{\varepsilon} \mid y\right) \geqslant \pi_{\Delta}\left(C_{\ell}^{\varepsilon} \mid y\right)\left[1-\varepsilon_{\ell} e^{2 v_{\ell}}\right]
$$

which can be iterated to the following one

$$
\pi_{\Delta}\left(C^{\varepsilon} \mid y\right) \geqslant \pi_{\Delta}\left(C_{\Delta}^{\varepsilon} \mid y\right) \prod_{\ell \in \Delta}\left[1-\varepsilon_{\ell} e^{2 v_{\ell}}\right]=\mathbb{I}_{C_{\Delta}^{\varepsilon}}(y) \prod_{\ell \in \Delta}\left[1-\varepsilon_{\ell} e^{2 v_{\ell}}\right]=\prod_{\ell \in \Delta}\left[1-\varepsilon_{\ell} e^{2 v_{\ell}}\right] \geqslant 1-\varepsilon .
$$

This yields the relative weak compactness of the sequence $\left\{\pi_{\Delta}(\cdot \mid y)\right\}_{\mathcal{D}}$ and hence completes the proof.

Along with the measures (1.9) we will use the following ones

$$
\nu_{\Lambda}\left(\mathrm{d} x_{\Lambda} \mid y\right)=\frac{1}{Z_{\Lambda}(y)} \exp \left[V_{\Lambda}\left(x_{\Lambda} \mid y\right)\right] \chi_{\Lambda}\left(\mathrm{d} x_{\Lambda}\right)
$$

Note that $\nu_{\Lambda}(\cdot \mid y) \in \mathcal{P}\left(X_{\Lambda}\right)$. A function $f: X \rightarrow \mathbb{R}$ is called local if it is measurable with respect to the $\sigma$-field (1.6). The smallest (in the sense of inclusion) $\Delta \in \mathfrak{L}_{\text {fin }}$ such that $f$ is $\mathcal{B}\left(X_{\Delta}\right)$-measurable is called the support of $f$. In this case

$$
\pi_{\Delta}(f \mid y)=\nu_{\Delta}(f \mid y)
$$

For $V=0$, every $\pi_{\Lambda}(\cdot \mid y)$ is independent of $y$ and the family $\left\{\pi_{\Lambda}\right\}_{\Lambda \in \mathfrak{L}_{\text {fin }}}$ is consistent in the Kolmogorov sense. Then by the Kolmogorov lemma, $\mathcal{G}(0)$ is a singleton. We are going to prove that, for sparse graphs, the set $\mathcal{G}(V)$ contains at most one element if $V$ is nonzero but $\|V\|$ is small. Here

$$
\|V\|=\sup _{\left\langle\ell, \ell^{\prime}\right\rangle \in \mathrm{E}}\left\|V_{\ell \ell^{\prime}}\right\|
$$

Set

$$
\varkappa(V)=16[\exp (4\|V\|)-1]
$$

Our main result is as follows:

Theorem 1.8. Let the graph be sparse. Then there exists $\varkappa_{*} \in(0,1)$ such that the set $\mathcal{G}(V)$ contains one element only if $\varkappa(V) \leqslant \varkappa_{*}$.

The proof is based on an extension and refinement of the Bassalygo-Dobrushin technique, developed below in a sequence of lemmas.

\section{The main lemma}

For $\left\langle\ell, \ell^{\prime}\right\rangle \in \mathrm{E}$, we set, c.f. (1.20),

$$
\varkappa_{\ell \ell^{\prime}}=16\left[\exp \left(4\left\|V_{\ell \ell^{\prime}}\right\|\right)-1\right] .
$$


Then, for a path $\vartheta\left(\ell, \ell^{\prime}\right)$, we define

$$
R\left[\vartheta\left(\ell, \ell^{\prime}\right)\right]=4^{\varsigma(\ell)+\varsigma\left(\ell^{\prime}\right)} \prod_{i=0}^{n-1} \varkappa_{\ell_{i} \ell_{i+1}}
$$

where $\varsigma(\ell)=-1$ if $n_{\ell}=1$ and $\varsigma(\ell)=0$ if $n_{\ell} \geqslant 2$. Finally, for $\Delta \in \mathfrak{L}_{\text {fin }}$, we set

$$
S_{\Delta}\left(\ell, \ell^{\prime}\right)=\sum R\left[\vartheta\left(\ell, \ell^{\prime}\right)\right]
$$

where the summation is performed over all self-avoiding paths in $\Delta$ connecting $\ell$ with $\ell^{\prime}$.

Definition 2.1. Given $\ell_{0} \in \Delta \cup \partial_{\mathrm{L}} \Delta$, a subset $\Lambda \subset \Delta \in \mathfrak{L}_{\text {fin }}$ is called proper for $\ell_{0}$ if

$$
\sum_{\ell \in \Lambda} S_{\Delta}\left(\ell, \ell_{0}\right) \leqslant 1
$$

Lemma 2.2 (Main). Let $\Lambda$ be proper in $\Delta$ for some $\ell_{0} \in \partial_{\mathrm{L}} \Delta$, and let $y, z \in X$ differ at this $\ell_{0}$ only. Then

$$
\left|\frac{\nu_{\Delta}\left(\mathrm{d} x_{\Lambda} \mid y\right)}{\nu_{\Delta}\left(\mathrm{d} x_{\Lambda} \mid z\right)}-1\right| \leqslant \sum_{\ell \in \Lambda} S_{\Delta}\left(\ell, \ell_{0}\right) .
$$

The proof of this lemma is based on the following technicalities. First, by the triangle inequality, it follows that, for $\alpha, \beta \in \mathbb{R}$,

$$
|\alpha \beta-1| \leqslant|\alpha-1|+|\beta-1|+|\alpha-1| \cdot|\beta-1| .
$$

Similarly, see Assertion 2 in [2], for any $n \in \mathbb{N}$ and real $\alpha_{1}, \ldots, \alpha_{n}$, one has

$$
\text { if } \quad \sum_{i=1}^{n}\left|\alpha_{i}\right| \leqslant 1, \quad \text { then } \quad\left|\prod_{i=1}^{n}\left(1+\alpha_{i}\right)-1\right| \leqslant 2 \sum_{i=1}^{n}\left|\alpha_{i}\right| .
$$

Let $(Y, \mathcal{B}(Y), P)$ be a probability space and $u, v$ be positive measurable functions on $Y$. Then, c.f. $(1.5)$,

$$
\left|\frac{\int u(y) P(\mathrm{~d} y)}{\int v(y) P(\mathrm{~d} y)}-1\right|=\left|\frac{P(u)}{P(v)}-1\right| \leqslant \sup _{y \in Y}\left|\frac{u(y)}{v(y)}-1\right| .
$$

Now let $u, v, w$ be positive measurable functions and $w$ be such that $P(w)=1$. Set

$$
M_{+}(u, v)=\max \left\{\sup _{y \in Y} u(y) ; \sup _{y \in Y} v(y)\right\}, \quad M_{-}(u, v)=\min \left\{\inf _{y \in Y} u(y) ; \inf _{y \in Y} v(y)\right\}
$$

and suppose that $M_{-}(u, v)>0$. Then

$$
\left|\frac{\int w(y) u(y) P(\mathrm{~d} y)}{\int v(y) P(\mathrm{~d} y)}-1\right| \leqslant \frac{M_{+}(u, v)}{M_{-}(u, v)}-1 .
$$

Let $y_{0} \in Y$ be such that

$$
v\left(y_{0}\right) \leqslant \int v(y) P(\mathrm{~d} y)
$$

which obviously exists. Suppose that there exist positive $\gamma, \varepsilon, \delta$, such that

$$
|w(y)-1| \leqslant \gamma, \quad\left|\frac{u(y)}{u\left(y_{0}\right)}-1\right| \leqslant \varepsilon, \quad\left|\frac{u(y)}{v(y)}-1\right| \leqslant \delta .
$$

Then

$$
\left|\frac{\int w(y) u(y) P(\mathrm{~d} y)}{\int v(y) P(\mathrm{~d} y)}-1\right| \leqslant \delta+\varepsilon \gamma+\delta \varepsilon \gamma .
$$


Let us prove this. Set

$$
\gamma(y)=w(y)-1 ; \quad \varepsilon(y)=\frac{u(y)}{u\left(y_{0}\right)}-1 ; \quad \delta(y)=\frac{u(y)}{v(y)}-1 .
$$

As $P(w)=1$, one has $P(\gamma)=0$. Moreover, $|\gamma(y)| \leqslant \gamma,|\varepsilon(y)| \leqslant \varepsilon$, and $|\delta(y)| \leqslant \delta$. Then

$$
\begin{aligned}
\frac{\int w(y) u(y) P(\mathrm{~d} y)}{\int v(y) P(\mathrm{~d} y)}= & \frac{1}{P(v)}\left[\int u(y) P(\mathrm{~d} y)+\int \gamma(y) u(y) P(\mathrm{~d} y)\right] \\
= & \frac{1}{P(v)}\left[\int v(y) P(\mathrm{~d} y)+\int v(y) \delta(y) P(\mathrm{~d} y)\right. \\
& \left.+\int \gamma(y) u\left(y_{0}\right) P(\mathrm{~d} y)+u\left(y_{0}\right) \int \gamma(y) \varepsilon(y) P(\mathrm{~d} y)\right] \\
= & 1+\int \delta(y) Q(\mathrm{~d} y)+\frac{u\left(y_{0}\right)}{v\left(y_{0}\right)} \cdot \frac{v\left(y_{0}\right)}{P(v)} \cdot \int \gamma(y) \varepsilon(y) P(\mathrm{~d} y)
\end{aligned}
$$

where $Q(\mathrm{~d} y)=v(y) P(\mathrm{~d} y) / P(v)$. Thereafter, the estimate $(2.11)$ is straightforward.

Proof of Lemma 2.2: The measure (1.18) can be written in the following form

$$
\begin{aligned}
\nu_{\Delta}\left(\mathrm{d} x_{\Delta} \mid y\right) & =\frac{1}{Z_{\Delta}(y)} \exp \left[V_{\Delta}\left(x_{\Delta}\right)+\sum_{\ell \in \Delta} V_{\ell \ell_{0}}\left(x_{\ell}, y_{\ell_{0}}\right)\right] \tilde{\chi}_{\Delta}\left(\mathrm{d} x_{\Delta}\right), \\
\tilde{\chi}_{\ell}\left(\mathrm{d} x_{\ell}\right) & =\exp \left[\sum_{\ell^{\prime} \in \partial_{\mathrm{L}} \Delta \backslash\left\{\ell_{0}\right\}} V_{\ell \ell^{\prime}}\left(x_{\ell}, y_{\ell^{\prime}}\right)\right] \chi_{\ell}\left(\mathrm{d} x_{\ell}\right) .
\end{aligned}
$$

Since the configuration on $\partial_{\mathrm{L}} \Delta \backslash\left\{\ell_{0}\right\}$ is going to be fixed by the very end of the proof and the concrete choice of the single-point measures plays no role, we can change $\chi$ 's to $\tilde{\chi}$ 's, which is equivalent to considering the case of $\partial_{\mathrm{L}} \Delta=\left\{\ell_{0}\right\}$. Therefore, in this section we deal with the following finite graph

$$
\mathrm{G} \stackrel{\text { def }}{=}\left(\Delta^{\text {aug }}, \mathrm{E}_{\Delta}^{\text {aug }}\right), \quad \Delta^{\text {aug }}=\Delta \cup\left\{\ell_{0}\right\}, \quad \mathrm{E}_{\Delta}^{\text {aug }}=\mathrm{E}_{\Delta} \cup \partial_{\mathrm{E}} \ell_{0} .
$$

The proof of the lemma is based on an inductive construction employing transformations of G. Given $n, q \in \mathbb{N}$, let $\mathfrak{G}_{n, q}$ be the family of all finite graphs such that $n_{\ell} \leqslant n$ for all vertices, and the number of the vertices of degree $n$ is $q$. We still suppose that the graphs have no loops, multiple edges, and isolated vertices. By $\mathfrak{G}_{2,0}$ we denote the family of minimal graphs, i.e., such that $n_{\ell}=1$ for all vertices. As we are going to compare different graphs, by the end of this section we write $\nu_{\mathrm{G}}$ and $S_{\mathrm{G}}$ instead of $\nu_{\Delta}$ and $S_{\Delta}$ respectively. Finally, for a set of vertices $\Lambda$ and $\ell \in \Lambda$, we write

$$
\Lambda_{\ell}=\Lambda \backslash\{\ell\}
$$

Given $G \in \mathfrak{G}_{2,0}$, let $\ell_{1}$ be the only neighbor of $\ell_{0}$. Then the projection of the measure (1.18) onto $\mathcal{B}\left(X_{\Lambda}\right)$ can be written in the form

$$
\begin{aligned}
& \nu_{\mathrm{G}}\left(\mathrm{d} x_{\Lambda} \mid y\right)=\frac{J\left(x_{\ell_{1}}, y\right)}{K(y)} \cdot \frac{F\left(x_{\Lambda_{\ell_{1}}}\right) \chi_{\Lambda_{\ell_{1}}}\left(\mathrm{~d} x_{\Lambda_{\ell_{1}}}\right)}{\int_{X_{\ell_{1}}} F\left(x_{\Lambda_{\ell_{1}}}\right) \chi_{\Lambda_{\ell_{1}}}\left(\mathrm{~d} x_{\Lambda_{\ell_{1}}}\right)}, \quad \ell_{1} \in \Lambda \\
& \nu_{\mathrm{G}}\left(\mathrm{d} x_{\Lambda} \mid y\right)=\frac{F\left(x_{\Lambda_{\ell}}\right) \chi_{\Lambda_{\ell}}\left(\mathrm{d} x_{\Lambda_{\ell}}\right)}{\int_{X_{\ell}} F\left(x_{\Lambda_{\ell}}\right) \chi_{\Lambda_{\ell}}\left(\mathrm{d} x_{\Lambda_{\ell}}\right)}, \quad \ell_{1} \in \Delta \backslash \Lambda .
\end{aligned}
$$

where

$$
J\left(x_{\ell_{1}}, y\right)=\exp \left[V_{\ell_{1} \ell_{0}}\left(x_{\ell_{1}}, y_{\ell_{0}}\right)\right], \quad K(y)=\int_{X_{\ell_{1}}} J\left(x_{\ell_{1}}, y\right) \chi_{\ell_{1}}\left(\mathrm{~d} x_{\ell_{1}}\right)
$$


Thereby,

$$
\frac{\nu_{\mathrm{G}}\left(\mathrm{d} x_{\Lambda} \mid y\right)}{\nu_{\mathrm{G}}\left(\mathrm{d} x_{\Lambda} \mid z\right)}=1
$$

if $\ell_{1} \in \Delta \backslash \Lambda$. Otherwise,

$$
\left|\frac{\nu_{\mathrm{G}}\left(\mathrm{d} x_{\Lambda} \mid y\right)}{\nu_{\mathrm{G}}\left(\mathrm{d} x_{\Lambda} \mid z\right)}-1\right| \leqslant \exp \left(4\left\|V_{\ell_{1} \ell_{0}}\right\|\right)-1=\frac{\varkappa_{\ell_{1} \ell_{0}}}{16}=S_{\mathrm{G}}\left(\ell_{1}, \ell_{0}\right)=\sum_{\ell \in \Lambda} S_{\mathrm{G}}\left(\ell, \ell_{0}\right) .
$$

Given $n, q \in \mathbb{N}$, suppose now that, for any $G \in \mathfrak{G}_{n, q}$ having the form (2.12), the property stated in Lemma 2.2 holds true. Let us show that this property holds also for any $\mathrm{G} \in \mathfrak{G}_{n, q+1}$. For such a graph, let $b$ be the vertex for which $n_{b}=n$. Then we introduce a new graph, $\widetilde{\mathrm{G}}$, obtained from $\mathrm{G}$ by unglueing the vertex $b$. This means that instead of $b, \widetilde{\mathrm{G}}$ has vertices $b_{1}, \ldots, b_{n}$, for which $n_{b_{i}}=1$, $i=1, \ldots, n$. Each of $b_{i}$ 's is adjacent to the corresponding $\ell_{i} \in \partial_{\mathrm{L}} b$. All other vertices and edges of $\mathrm{G}$ and $\widetilde{\mathrm{G}}$ coincide. Thus, the new vertex set is $\tilde{\Delta}^{\text {aug }}=\Delta_{b}^{\text {aug }} \cup \Gamma$, where $\Gamma=\left\{b_{1}, \ldots, b_{n}\right\}$. Therefore, $\widetilde{\mathrm{G}} \in \mathfrak{G}_{n, q}$.

The following three cases: $b \in \Lambda, b=\ell_{0}, b \in \Delta \backslash \Lambda$, will be considered separately.

Case I: $b \in \Lambda$.

Here $\tilde{\Delta}=\Delta_{b} \cup \Gamma, \tilde{\Lambda}=\Lambda_{b} \cup \Gamma$. We set, c.f. (1.18) and (2.13),

$$
\nu_{\widetilde{\mathrm{G}}}\left(\mathrm{d} x_{\tilde{\Delta}} \mid y\right)=\frac{1}{Z_{\tilde{\Delta}}(y)} \exp \left[V_{\Delta_{b}}\left(x_{\Delta_{b}} \mid y\right)+\sum_{i=1}^{n} V_{\ell_{i}, b}\left(x_{\ell_{i}}, x_{b_{i}}\right)\right] \chi_{\Delta_{b}}\left(\mathrm{~d} x_{\Delta_{b}}\right) \chi_{\Gamma}\left(\mathrm{d} x_{\Gamma}\right)
$$

where

$$
\chi_{\Gamma}\left(\mathrm{d} x_{\Gamma}\right) \stackrel{\text { def }}{=} \bigotimes_{\ell \in \Gamma} \chi_{b}\left(\mathrm{~d} x_{\ell}\right)=\bigotimes_{i=1}^{n} \chi_{b}\left(\mathrm{~d} x_{b_{i}}\right) .
$$

Here we consider the case where $b$ and $\ell_{0}$ are not adjacent. The construction in the opposite case is quite similar. The projection of the measure $(2.15)$ onto $\mathcal{B}\left(X_{\tilde{\Lambda}}\right)$ can be written in the form

$$
\nu_{\widetilde{\mathrm{G}}}\left(\mathrm{d} x_{\tilde{\Lambda}} \mid y\right)=\nu_{\widetilde{\mathrm{G}}}\left(\mathrm{d}\left(x_{\Lambda_{b}} \times x_{\Gamma}\right) \mid y\right)=F\left(x_{\Lambda_{b}} \times x_{\Gamma} \mid y\right) \chi_{\Lambda_{b}}\left(\mathrm{~d} x_{\Lambda_{b}}\right) \chi_{\Gamma}\left(\mathrm{d} x_{\Gamma}\right)
$$

whereas its projection onto $\mathcal{B}\left(X_{\Gamma}\right)$ is

$$
\nu_{\widetilde{\mathrm{G}}}\left(\mathrm{d} x_{\Gamma} \mid y\right)=\Phi\left(x_{b_{1}}, \ldots x_{b_{n}} \mid y\right) \chi_{\Gamma}\left(\mathrm{d} x_{\Gamma}\right)
$$

where

$$
\Phi\left(x_{b_{1}}, \ldots x_{b_{n}} \mid y\right) \stackrel{\text { def }}{=} \int_{X_{\Lambda_{b}}} F\left(x_{\Lambda_{b}} \times x_{\Gamma} \mid y\right) \chi_{\Lambda_{b}}\left(\mathrm{~d} x_{\Lambda_{b}}\right) .
$$

With the help of these functions, the projection of the 'old' measure onto $\mathcal{B}\left(X_{\Lambda}\right)$ can be written

$$
\nu_{\mathrm{G}}\left(\mathrm{d} x_{\Lambda} \mid y\right)=\frac{F\left(x_{\Lambda_{b}} \times\left(x_{b} \times \cdots \times x_{b}\right) \mid y\right)}{\int_{X_{b}} \Phi\left(x_{b}, \ldots, x_{b} \mid y\right) \chi_{b}\left(\mathrm{~d} x_{b}\right)} \cdot \chi_{\Lambda_{b}}\left(\mathrm{~d} x_{\Lambda_{b}}\right) \chi_{b}\left(\mathrm{~d} x_{b}\right) .
$$

Therefore,

$$
\frac{\nu_{\mathrm{G}}\left(\mathrm{d} x_{\Lambda} \mid y\right)}{\nu_{\mathrm{G}}\left(\mathrm{d} x_{\Lambda} \mid z\right)}=\frac{F\left(x_{\Lambda_{b}} \times\left(x_{b} \times \cdots \times x_{b}\right) \mid y\right)}{F\left(x_{\Lambda_{b}} \times\left(x_{b} \times \cdots \times x_{b}\right) \mid z\right)} \frac{\int_{X_{b}} \Phi\left(x_{b}, \ldots, x_{b} \mid z\right) \chi_{b}\left(\mathrm{~d} x_{b}\right)}{\int_{X_{b}} \Phi\left(x_{b}, \ldots, x_{b} \mid y\right) \chi_{b}\left(\mathrm{~d} x_{b}\right)}
$$

Now by means of $(2.6)$ one gets

$$
\left|\frac{\nu_{\mathrm{G}}\left(\mathrm{d} x_{\Lambda} \mid y\right)}{\nu_{\mathrm{G}}\left(\mathrm{d} x_{\Lambda} \mid z\right)}-1\right| \leqslant A+B+A \cdot B
$$


where

$$
\begin{aligned}
A & =\left|\frac{F\left(x_{\Lambda_{b}} \times\left(x_{b} \times \cdots \times x_{b}\right) \mid y\right)}{F\left(x_{\Lambda_{b}} \times\left(x_{b} \times \cdots \times x_{b}\right) \mid z\right)}-1\right| \leqslant \sup _{x_{b_{1}}, \ldots, x_{b_{n}} \in X_{b}}\left|\frac{F\left(x_{\Lambda_{b}} \times\left(x_{b_{1}} \times \cdots \times x_{b_{n}}\right) \mid y\right)}{F\left(x_{\Lambda_{b}} \times\left(x_{b_{1}} \times \cdots \times x_{b_{n}}\right) \mid z\right)}-1\right| \\
& =\sup _{x_{b_{1}}, \ldots, x_{b_{n}} \in X_{b}}\left|\frac{\nu_{\widetilde{\mathrm{G}}}\left(\mathrm{d} x_{\tilde{\Lambda}} \mid y\right)}{\nu_{\widetilde{\mathrm{G}}}\left(\mathrm{d} x_{\tilde{\Lambda}} \mid z\right)}-1\right| .
\end{aligned}
$$

and

$$
B=\left|\frac{\int_{X_{b}} \Phi\left(x_{b}, \ldots, x_{b} \mid z\right) \chi_{b}\left(\mathrm{~d} x_{b}\right)}{\int_{X_{b}} \Phi\left(x_{b}, \ldots, x_{b} \mid y\right) \chi_{b}\left(\mathrm{~d} x_{b}\right)}-1\right| .
$$

By (2.8), it follows that

$$
\begin{aligned}
B & \leqslant \sup _{x_{b} \in X_{b}}\left|\frac{\Phi\left(x_{b}, \ldots, x_{b} \mid z\right)}{\Phi\left(x_{b}, \ldots, x_{b} \mid y\right)}-1\right| \leqslant \sup _{x_{b_{1}}, \ldots, x_{b_{n}} \in X_{b}}\left|\frac{\Phi\left(x_{b_{1}}, \ldots, x_{b_{n}} \mid z\right)}{\Phi\left(x_{b_{1}}, \ldots, x_{b_{n}} \mid y\right)}-1\right| \\
& =\sup _{x_{b_{1}}, \ldots, x_{b_{n}} \in X_{b}}\left|\frac{\nu_{\widetilde{\mathrm{G}}}\left(\mathrm{d} x_{\tilde{\Gamma}} \mid y\right)}{\nu_{\widetilde{\mathrm{G}}}\left(\mathrm{d} x_{\tilde{\Gamma}} \mid z\right)}-1\right| .
\end{aligned}
$$

In order to proceed further in estimating $A$ and $B$ let us compare $S_{\widetilde{G}}\left(\ell, \ell_{0}\right)$ with $S_{\mathrm{G}}\left(\ell, \ell_{0}\right)$. As the potentials $V_{\ell \ell^{\prime}}$ for $\ell, \ell^{\prime} \in \Delta_{b}^{\text {aug }}$ are the same in both measures (1.18) and (2.15), the quantities under comparing are calculated by $(2.2),(2.3)$ with one and the same $\varkappa_{\ell \ell^{\prime}}$. Furthermore, by (2.15) it follows that

$$
\varkappa_{b_{i} \ell_{i}}=\varkappa_{b \ell_{i}}, \quad i=1, \ldots n .
$$

Thus, taking into account that $n_{b_{i}}=1, i=1, \ldots, n$ and $n_{b}>1$, one gets, see $(2.2)$

$$
\sum_{i=1}^{n} S_{\widetilde{\mathrm{G}}}\left(b_{i}, \ell_{0}\right)=S_{\mathrm{G}}\left(b, \ell_{0}\right) / 4 .
$$

Furthermore, every self-avoiding path $\vartheta\left(\ell, \ell_{0}\right)$ in $\tilde{\Delta}^{\text {aug }}$ which starts at $\ell \in \Lambda_{b}$ always avoids $\Gamma$. Therefore,

$$
S_{\widetilde{\mathrm{G}}}\left(\ell, \ell_{0}\right)=S_{\mathrm{G}}^{b}\left(\ell, \ell_{0}\right) \leqslant S_{\mathrm{G}}\left(\ell, \ell_{0}\right), \quad \text { for any } \ell \in \Lambda_{b} \text {. }
$$

Here and in the sequel, for $a \in \Delta^{\text {aug }}$, by $S_{\mathrm{G}}^{a}\left(\ell, \ell^{\prime}\right)$ we denote the quantity calculated according to (2.3) with the summation taken over all paths which avoid $a$. The latter two estimates yield

$$
\sum_{\ell \in \tilde{\Lambda}} S_{\widetilde{\mathrm{G}}}\left(\ell, \ell_{0}\right) \leqslant \sum_{\ell \in \Lambda} S_{\mathrm{G}}\left(\ell, \ell_{0}\right)
$$

hence, $\tilde{\Lambda}$ is proper in $\tilde{\Delta}$ for $\ell_{0}$ due to the same property of $\Lambda$ in $\Delta$ with respect to $\ell_{0}$. Hence, as a subset of $\tilde{\Lambda}$, by (2.25) $\Gamma$ is also proper in $\tilde{\Delta}$ for $\ell_{0}$. Thereafter, by the inductive assumption and (2.23), (2.24) we get

$$
\begin{aligned}
A & \leqslant \sum_{\ell \in \Lambda_{b}} S_{\widetilde{\mathrm{G}}}\left(\ell, \ell_{0}\right)+\sum_{i=1}^{n} S_{\widetilde{\mathrm{G}}}\left(b_{i}, \ell_{0}\right) \leqslant 1, \\
B & \leqslant \sum_{i=1}^{n} S_{\widetilde{\mathrm{G}}}\left(b_{i}, \ell_{0}\right) \leqslant 1 .
\end{aligned}
$$


Now we apply these estimates in (2.22) and obtain

$$
\begin{aligned}
\left|\frac{\nu_{\mathrm{G}}\left(\mathrm{d} x_{\Lambda} \mid y\right)}{\nu_{\mathrm{G}}\left(\mathrm{d} x_{\Lambda} \mid z\right)}-1\right| \leqslant & \sum_{\ell \in \Lambda_{b}} S_{\widetilde{\mathrm{G}}}\left(\ell, \ell_{0}\right)+\sum_{i=1}^{n} S_{\widetilde{\mathrm{G}}}\left(b_{i}, \ell_{0}\right)+\sum_{i=1}^{n} S_{\widetilde{\mathrm{G}}}\left(b_{i}, \ell_{0}\right) \\
& \quad+\left(\sum_{\ell \in \Lambda_{b}} S_{\widetilde{\mathrm{G}}}\left(\ell, \ell_{0}\right)+\sum_{i=1}^{n} S_{\widetilde{\mathrm{G}}}\left(b_{i}, \ell_{0}\right)\right) \cdot \sum_{i=1}^{n} S_{\widetilde{\mathrm{G}}}\left(b_{i}, \ell_{0}\right) \\
\leqslant & \sum_{\ell \in \Lambda_{b}} S_{\widetilde{\mathrm{G}}}\left(\ell, \ell_{0}\right)+3 \sum_{i=1}^{n} S_{\widetilde{\mathrm{G}}}\left(b_{i}, \ell_{0}\right) \\
\leqslant & \sum_{\ell \in \Lambda_{b}} S_{\mathrm{G}}\left(\ell, \ell_{0}\right)+\frac{3}{4} S_{\mathrm{G}}\left(b, \ell_{0}\right) \leqslant \sum_{\ell \in \Lambda} S_{\mathrm{G}}\left(\ell, \ell_{0}\right) \leqslant 1,
\end{aligned}
$$

which complete the proof for this case.

Case II: $b=\ell_{0}$.

Now $\Delta=\tilde{\Delta}$ and $\tilde{\Delta}^{\text {aug }}=\Delta \cup \Gamma$, where as above $\Gamma=\left\{b_{1}, \ldots, b_{n}\right\}$. Since we can compare the measures with boundary conditions which differ at one point only, we introduce

$$
\begin{aligned}
\nu_{\widetilde{\mathrm{G}}}^{(j)}\left(\mathrm{d} x_{\Delta} \mid y, z\right)= & \frac{1}{Z_{\widetilde{\mathrm{G}}}^{(j)}(y, z)} \exp \left[\sum_{\left\langle\ell, \ell^{\prime}\right\rangle \in \mathrm{E}_{\Delta}} V_{\ell \ell^{\prime}}\left(x_{\ell}, x_{\ell^{\prime}}\right)\right. \\
& \left.+\sum_{i=1}^{j} V_{\ell_{i} \ell_{0}}\left(x_{\ell_{i}}, z_{b_{i}}\right)+\sum_{i=j+1}^{n} V_{\ell_{i} \ell_{0}}\left(x_{\ell_{i}}, y_{b_{i}}\right)\right] \chi_{\Delta}\left(\mathrm{d} x_{\Delta}\right),
\end{aligned}
$$

where $j=0, \ldots, n, y_{b_{i}}=y_{\ell_{0}}, z_{b_{i}}=z_{\ell_{0}}$, for all $i=1, \ldots, n$, and $y_{\ell_{0}}, z_{\ell_{0}}$ are the same as in $(2.5)$. Thereby, we have

$$
\frac{\nu_{\mathrm{G}}\left(\mathrm{d} x_{\Lambda} \mid y\right)}{\nu_{\mathrm{G}}\left(\mathrm{d} x_{\Lambda} \mid z\right)}=\prod_{i=1}^{n} \frac{\nu_{\widetilde{\mathrm{G}}}^{(i-1)}\left(\mathrm{d} x_{\Delta} \mid y, z\right)}{\nu_{\widetilde{\mathrm{G}}}^{(i)}\left(\mathrm{d} x_{\Delta} \mid y, z\right)}
$$

The boundary conditions for $\nu_{\widetilde{\mathrm{G}}}^{(i-1)}$ and $\nu_{\widetilde{\mathrm{G}}}^{(i)}$ differ at the vertex $b_{i}$, the degree of which in $\widetilde{\mathrm{G}}$ is 1 . Then, similarly to (2.25), we have that for any $\ell \in \Lambda$,

$$
\sum_{i=1}^{n} S_{\widetilde{\mathrm{G}}}\left(\ell, b_{i}\right)=S_{\mathrm{G}}\left(\ell, \ell_{0}\right) / 4 \text {. }
$$

Therefore, $\Lambda$ is proper in $\tilde{\Delta}$ for every $b_{i}, i=1, \ldots, n$. Thus, by the inductive assumption, it follows that

$$
\left|\frac{\nu_{\widetilde{\mathrm{G}}}^{(i-1)}\left(\mathrm{d} x_{\Delta} \mid y, z\right)}{\nu_{\widetilde{\mathrm{G}}}^{(i)}\left(\mathrm{d} x_{\Delta} \mid y, z\right)}-1\right| \leqslant \sum_{\ell \in \Lambda} S_{\widetilde{\mathrm{G}}}\left(\ell, b_{i}\right), \quad \text { for all } i=1, \ldots, n
$$

Now we apply this estimate in (2.29) together with (2.7), (2.30) and obtain

$$
\left|\frac{\nu_{\mathrm{G}}\left(\mathrm{d} x_{\Lambda} \mid y\right)}{\nu_{\mathrm{G}}\left(\mathrm{d} x_{\Lambda} \mid z\right)}-1\right| \leqslant 2 \sum_{i=1}^{n} \sum_{\ell \in \Lambda} S_{\widetilde{\mathrm{G}}}\left(\ell, b_{i}\right) \leqslant \frac{1}{2} \sum_{\ell \in \Lambda} S_{\mathrm{G}}\left(\ell, \ell_{0}\right),
$$

which completes the proof for this case.

Case III: $b \in \Delta \backslash \Lambda$.

By (2.3), it follows that

$$
\sum_{\ell \in \Lambda} S_{\mathrm{G}}\left(\ell, \ell_{0}\right)=\sum_{\ell \in \Lambda} S_{\mathrm{G}}^{b}\left(\ell, \ell_{0}\right)+\left(\sum_{\ell \in \Lambda} S_{\mathrm{G}}(\ell, b)\right) \cdot S_{\mathrm{G}}\left(b, \ell_{0}\right) .
$$


The following three cases of the location of $b$ will be considered separately:
(a) $b$ is far from both $\ell_{0}$ and $\Lambda$;
(b) $\quad b$ is close to $\ell_{0}$ and far from $\Lambda$;
(c) $b$ is far from $\ell_{0}$ and close to $\Lambda$;

\section{Subcase III a:}

Here

$$
S_{\mathrm{G}}\left(b, \ell_{0}\right) \leqslant 1 \quad \text { and } \quad \sum_{\ell \in \Lambda} S_{\mathrm{G}}(\ell, b) \leqslant 1 .
$$

In contrast to the cases considered above, we set $\tilde{\Delta}=\Delta_{b}$ and $\tilde{\Delta}^{\text {aug }}=\Delta_{b} \cup\left\{b, \ell_{0}\right\}$. Thereby,

$$
\nu_{\widetilde{\mathrm{G}}}\left(\mathrm{d} x_{\Delta_{b}} \mid x_{b}, y\right)=\frac{1}{Z_{\widetilde{\mathrm{G}}}\left(x_{b}, y\right)} \exp \left[V_{\Delta_{b}}\left(x_{\Delta_{b}} \mid y\right)+\sum_{\ell \in \Delta_{b}} V_{\ell b}\left(x_{\ell}, x_{b}\right)\right] \chi_{\Delta_{b}}\left(\mathrm{~d} x_{\Delta_{b}}\right),
$$

which by (1.19) and (1.15) yields

$$
\nu_{\mathrm{G}}\left(\mathrm{d} x_{\Delta} \mid y\right)=\int_{X_{\Delta}} \nu_{\widetilde{\mathrm{G}}}\left(\mathrm{d} x_{\Delta_{b}} \mid \xi_{b}, y\right) \nu_{\mathrm{G}}\left(\mathrm{d} \xi_{\Delta} \mid y\right)=\int_{X_{b}} \nu_{\widetilde{\mathrm{G}}}\left(\mathrm{d} x_{\Delta_{b}} \mid x_{b}, y\right) \nu_{\mathrm{G}}\left(\mathrm{d} x_{b} \mid y\right) .
$$

For any $\Lambda \subset \Delta_{b}$, the projection of the measure (2.34) onto $\mathcal{B}\left(X_{\Lambda}\right)$ can be written in the form

$$
\nu_{\widetilde{\mathrm{G}}}\left(\mathrm{d} x_{\Lambda} \mid x_{b}, y\right)=F\left(x_{\Lambda} \mid x_{b}, y\right) \chi_{\Lambda}\left(\mathrm{d} x_{\Lambda}\right)
$$

where $F$ is a positive continuous function of all its arguments. Therefore,

$$
\frac{\nu_{\mathrm{G}}\left(\mathrm{d} x_{\Lambda} \mid y\right)}{\nu_{\mathrm{G}}\left(\mathrm{d} x_{\Lambda} \mid z\right)}=\frac{\int_{X_{b}} F\left(x_{\Lambda} \mid x_{b}, y\right) \nu_{\mathrm{G}}\left(\mathrm{d} x_{b} \mid y\right)}{\int_{X_{b}} F\left(x_{\Lambda} \mid x_{b}, z\right) \nu_{\mathrm{G}}\left(\mathrm{d} x_{b} \mid z\right)}
$$

The right-hand side of the latter is of the type of the left-hand side of (2.11) and hence can be correspondingly estimated. By $(2.33)$, the set $\{b\}$ is proper for $\ell_{0}$; hence, as was established in Case I,

$$
\left|\frac{\nu_{\mathrm{G}}\left(\mathrm{d} x_{b} \mid y\right)}{\nu_{\mathrm{G}}\left(\mathrm{d} x_{b} \mid z\right)}-1\right| \leqslant S_{\mathrm{G}}\left(b, \ell_{0}\right) \stackrel{\text { def }}{=} \gamma .
$$

By $(2.34),(2.36)$, it follows that

$$
\frac{\nu_{\tilde{\mathrm{G}}}\left(\mathrm{d} x_{\Lambda} \mid x_{b}, y\right)}{\nu_{\tilde{\mathrm{G}}}\left(\mathrm{d} x_{\Lambda} \mid x_{b}, y\right)}=\frac{F\left(x_{\Lambda} \mid x_{b}, y\right)}{F\left(x_{\Lambda} \mid x_{b}, z\right)}
$$

where the configuration $x_{b}$ can be treated as a parameter, see the very beginning of the proof of the lemma. Therefore, in this expression one can redefine $\tilde{G}$ by setting $\tilde{\Delta}^{\text {aug }}=\Delta_{b} \cup\left\{\ell_{0}\right\}$. Thus, for every $\ell \in \Delta_{b}$, one has

$$
S_{\widetilde{\mathrm{G}}}\left(\ell, \ell_{0}\right)=S_{\mathrm{G}}^{b}\left(\ell, \ell_{0}\right)
$$

which yields

$$
\left|\frac{F\left(x_{\Lambda} \mid x_{b}, y\right)}{F\left(x_{\Lambda} \mid x_{b}, z\right)}-1\right|=\left|\frac{\nu_{\widetilde{\mathrm{G}}}\left(\mathrm{d} x_{\Lambda} \mid x_{b}, y\right)}{\nu_{\widetilde{\mathrm{G}}}\left(\mathrm{d} x_{\Lambda} \mid x_{b}, z\right)}-1\right| \leqslant \sum_{\ell \in \Lambda} S_{\widetilde{\mathrm{G}}}\left(\ell, \ell_{0}\right)=\sum_{\ell \in \Lambda} S_{\mathrm{G}}^{b}\left(\ell, \ell_{0}\right) \stackrel{\text { def }}{=} \delta .
$$

Afterwards, one employs the inductive assumption, by which $\delta \leqslant 1$. Finally, we set

$$
\varepsilon=\frac{1}{2} \sum_{\ell \in \Lambda} S_{\mathrm{G}}(\ell, b)
$$


Furthermore, as in (2.31) we get

$$
\left|\frac{F\left(x_{\Lambda} \mid x_{b}, y\right)}{F\left(x_{\Lambda} \mid \xi_{b}, y\right)}-1\right|=\left|\frac{\nu_{\widetilde{\mathrm{G}}}\left(\mathrm{d} x_{\Lambda} \mid x_{b}, y\right)}{\nu_{\widetilde{\mathrm{G}}}\left(\mathrm{d} x_{\Lambda} \mid \xi_{b}, y\right)}-1\right| \leqslant \frac{1}{2} \sum_{\ell \in \Lambda} S_{\mathrm{G}}(\ell, b)
$$

holding for any $x_{b}, \xi_{b} \in X_{b}$. Finally, we pick $\xi_{b} \in X_{b}$ such that

$$
F\left(x_{\Lambda} \mid \xi_{b}, y\right) \leqslant \int_{X_{b}} F\left(x_{\Lambda} \mid x_{b}, y\right) \nu_{\mathrm{G}}\left(\mathrm{d} x_{b} \mid y\right)
$$

Thereby, we fix $x_{\Lambda} \in X_{\Lambda}, y_{\ell_{0}}, z_{\ell_{0}} \in X_{\ell_{0}}$ and obtain by (2.11) that

$$
\begin{aligned}
\left|\frac{\nu_{\mathrm{G}}\left(\mathrm{d} x_{\Lambda} \mid y\right)}{\nu_{\mathrm{G}}\left(\mathrm{d} x_{\Lambda} \mid z\right)}-1\right|= & \left|\frac{\int_{X_{b}} F\left(x_{\Lambda} \mid x_{b}, y\right) \nu_{\mathrm{G}}\left(\mathrm{d} x_{b} \mid y\right)}{\int_{X_{b}} F\left(x_{\Lambda} \mid x_{b}, z\right) \nu_{\mathrm{G}}\left(\mathrm{d} x_{b} \mid z\right)}-1\right| \leqslant \sum_{\ell \in \Lambda} S_{\mathrm{G}}^{b}\left(\ell, \ell_{0}\right)+\frac{1}{2} \sum_{\ell \in \Lambda} S_{\mathrm{G}}(\ell, b) S_{\mathrm{G}}\left(b, \ell_{0}\right) \\
& \leqslant \frac{1}{2} \sum_{\ell \in \Lambda} S_{\mathrm{G}}(\ell, b) S_{\mathrm{G}}\left(b, \ell_{0}\right) \cdot\left(\sum_{\ell \in \Lambda} S_{\mathrm{G}}^{b}\left(\ell, \ell_{0}\right)\right) \\
& \leqslant \sum_{\ell \in \Lambda} S_{\mathrm{G}}^{b}\left(\ell, \ell_{0}\right)+\sum_{\ell \in \Lambda} S_{\mathrm{G}}(\ell, b) S_{\mathrm{G}}\left(b, \ell_{0}\right)=\sum_{\ell \in \Lambda} S_{\mathrm{G}}\left(\ell, \ell_{0}\right)
\end{aligned}
$$

which completes the proof for this subcase.

\section{Subcase III b:}

Here

$$
\sum_{\ell \in \Lambda} S_{\mathrm{G}}\left(b, \ell_{0}\right)>1
$$

Therefore,

$$
\sum_{\ell \in \Lambda} S_{\mathrm{G}}^{b}\left(\ell, \ell_{0}\right)+\sum_{\ell \in \Lambda} S_{\mathrm{G}}(\ell, b)<\sum_{\ell \in \Lambda} S_{\mathrm{G}}^{b}\left(\ell, \ell_{0}\right)+\sum_{\ell \in \Lambda} S_{\mathrm{G}}(\ell, b) S_{\mathrm{G}}\left(b, \ell_{0}\right)=\sum_{\ell \in \Lambda} S_{\mathrm{G}}\left(\ell, \ell_{0}\right) \leqslant 1 .
$$

As above, we fix $x_{\Lambda} \in X_{\Lambda}, y_{\ell_{0}}, z_{\ell_{0}} \in X_{\ell_{0}}$, take arbitrarily $\xi_{b} \in X_{b}$, and introduce the following functions

$$
\Phi\left(x_{b}\right)=\frac{F\left(x_{\Lambda} \mid x_{b}, y\right)}{F\left(x_{\Lambda} \mid \xi_{b}, y\right)}, \quad \Psi\left(x_{b}\right)=\frac{F\left(x_{\Lambda} \mid x_{b}, z\right)}{F\left(x_{\Lambda} \mid \xi_{b}, y\right)}=\frac{F\left(x_{\Lambda} \mid x_{b}, z\right)}{F\left(x_{\Lambda} \mid x_{b}, y\right)} \Phi\left(x_{b}\right) .
$$

Thereby

$$
\left|\frac{\nu_{\mathrm{G}}\left(\mathrm{d} x_{\Lambda} \mid y\right)}{\nu_{\mathrm{G}}\left(\mathrm{d} x_{\Lambda} \mid z\right)}-1\right|=\left|\frac{\int_{X_{b}} F\left(x_{\Lambda} \mid x_{b}, y\right) \nu_{\mathrm{G}}\left(\mathrm{d} x_{b} \mid y\right)}{\int_{X_{b}} F\left(x_{\Lambda} \mid \xi_{b}, z\right) \nu_{\mathrm{G}}\left(\mathrm{d} \xi_{b} \mid z\right)}-1\right| \leqslant \sup _{x_{b} \in X_{b}}\left|\Psi\left(x_{b}\right)-1\right| .
$$

The latter estimate has been obtained by means of (2.9). But by (2.6), (2.40), (2.42), and (2.44), we get

$$
\begin{aligned}
\sup _{x_{b} \in X_{b}}\left|\Psi\left(x_{b}\right)-1\right| & \leqslant \frac{1}{2} \sum_{\ell \in \Lambda} S_{\mathrm{G}}(\ell, b)+\sum_{\ell \in \Lambda} S_{\mathrm{G}}^{b}\left(\ell, \ell_{0}\right)+\frac{1}{2} \sum_{\ell \in \Lambda} S_{\mathrm{G}}(\ell, b) \cdot\left(\sum_{\ell \in \Lambda} S_{\mathrm{G}}^{b}\left(\ell, \ell_{0}\right)\right) \\
& \leqslant \sum_{\ell \in \Lambda} S_{\mathrm{G}}^{b}\left(\ell, \ell_{0}\right)+\sum_{\ell \in \Lambda} S_{\mathrm{G}}(\ell, b)<\sum_{\ell \in \Lambda} S_{\mathrm{G}}\left(\ell, \ell_{0}\right),
\end{aligned}
$$

which completes the proof for this subcase.

\section{Subcase III c:}

Here

$$
\sum_{\ell \in \Lambda} S_{\mathrm{G}}(\ell, b)>1
$$


Then

$$
\sum_{\ell \in \Lambda} S_{\mathrm{G}}^{b}\left(\ell, \ell_{0}\right)+S_{\mathrm{G}}\left(b, \ell_{0}\right)<\sum_{\ell \in \Lambda} S_{\mathrm{G}}^{b}\left(\ell, \ell_{0}\right)+\sum_{\ell \in \Lambda} S_{\mathrm{G}}(\ell, b) S_{\mathrm{G}}\left(b, \ell_{0}\right)=\sum_{\ell \in \Lambda} S_{\mathrm{G}}\left(\ell, \ell_{0}\right) \leqslant 1 .
$$

Thereby, the set $\Lambda \cup\{b\}$ is proper for $\ell_{0}$ and hence can be considered as in Case I, which yields

$$
\frac{\nu_{\mathrm{G}}\left(\mathrm{d} x_{\Lambda} \times \mathrm{d} x_{b} \mid y\right)}{\nu_{\mathrm{G}}\left(\mathrm{d} x_{\Lambda} \times \mathrm{d} x_{b} \mid z\right)} \leqslant \sum_{\ell \in \Lambda} S_{\mathrm{G}}^{b}\left(\ell, \ell_{0}\right)+S_{\mathrm{G}}\left(b, \ell_{0}\right) .
$$

But

$$
\frac{\nu_{\mathrm{G}}\left(\mathrm{d} x_{\Lambda} \mid y\right)}{\nu_{\mathrm{G}}\left(\mathrm{d} x_{\Lambda} \mid z\right)}=\frac{\int_{X_{b}} \nu_{\mathrm{G}}\left(\mathrm{d} x_{\Lambda^{b}} \mid y\right)}{\int_{X_{b}} \nu_{\mathrm{G}}\left(\mathrm{d} x_{\Lambda^{b}} \mid z\right)}
$$

Thus, the estimate (2.5) is obtained from (2.48), (2.47) by means of (2.8).

In what follows, we have proven that the estimate (2.5) holds for proper subsets $\Lambda$ for any $\mathrm{G} \in \mathfrak{G}_{n, q}$ if it holds for $\mathrm{G} \in \mathfrak{G}_{n, 1}$. In the case $\mathrm{G} \in \mathfrak{G}_{n+1,1}$, one splits the only vertex of degree $n+1$ and obtains a graph in $\mathfrak{G}_{n, q}$, for which the property in question holds true.

\section{The proof of Theorem 1.8}

The proof of Theorem 1.8 will be based on Lemma 2.2 and on the property of $\mathcal{G}^{\text {ex }}$ described by Proposition 1.6. We begin, however, by proving another lemma.

Lemma 3.1. Suppose that, for every $\ell$, there exists a cofinal sequence $\left\{\Delta_{n}\right\}_{n \in \mathbb{N}}$, such that

$$
\lim _{n \rightarrow \infty} \sum_{\ell^{\prime} \in \partial_{\mathrm{L}} \Delta_{n}} S_{\Delta_{n}}\left(\ell, \ell^{\prime}\right)=0 .
$$

Then $\left|\mathcal{G}^{\mathrm{ex}}\right|=1$.

Proof. Let $C_{\mathrm{b}}^{\mathrm{loc}}(X)$ be the set of all bounded local continuous functions $f: X \rightarrow \mathbb{R}$. It is a measure defining class, which means that if for given $\mu, \tilde{\mu} \in \mathcal{P}(X)$, one has $\mu(f)=\tilde{\mu}(f)$ for all $f \in C_{\mathrm{b}}^{\mathrm{loc}}(X)$, then $\mu=\tilde{\mu}$. The proof of the lemma will be done by showing that if $\mu, \tilde{\mu} \in \mathcal{G}^{\mathrm{ex}}(V)$, then

$$
\mu(f)=\tilde{\mu}(f) \quad \text { for all } f \in C_{\mathrm{b}}^{\mathrm{loc}}(X) .
$$

Given $f \in C_{\mathrm{b}}^{\text {loc }}(X)$, let $\Lambda$ be its support. Then we pick the cofinal sequence $\left\{\Delta_{n}\right\}_{n \in \mathbb{N}}$ such that (3.1) and the following

$$
\pi_{\Delta_{n}}(\cdot \mid y) \Rightarrow \mu, \quad \pi_{\Delta_{n}}(\cdot \mid \tilde{y}) \Rightarrow \tilde{\mu}, \quad n \rightarrow+\infty,
$$

hold true for all $y \in A, \tilde{y} \in \tilde{A}$, such that $\mu(A)=1$ and $\tilde{\mu}(\tilde{A})=1$, see Proposition 1.6. For chosen $f$ and $\Delta_{n}$, such that $\Lambda \subset \Delta_{n}$, we have, see (1.19),

$$
\pi_{\Delta_{n}}(f \mid y)=\int_{X_{\Lambda}} f(x) \nu_{\Delta_{n}}\left(\mathrm{~d} x_{\Lambda} \mid y\right)
$$

Let $n \in \mathbb{N}$ be such that $\Lambda$ is proper in $\Delta_{n}$ for any $\ell \in \partial_{\mathrm{L}} \Delta_{n}$, see (3.1). Then we fix this $n$ and consider the set $\left\{\ell_{1}, \ldots, \ell_{N}\right\}=\partial_{\mathrm{L}} \Delta_{n}$. For $j=0,1, \ldots, N$, we introduce $y^{(j)} \in X$ as follows

$$
y_{\ell_{1}}^{(j)}=y_{\ell_{1}}, \ldots, y_{\ell_{N-j}}^{(j)}=y_{\ell_{N-j}}, y_{\ell_{N-j+1}}^{(j)}=\tilde{y}_{\ell_{N-j+1}}, \ldots, y_{N}^{(j)}=\tilde{y}_{\ell_{N}} .
$$

Thereafter, by $(2.7),(2.8),(3.3)$, and $(2.5)$, we get

$$
\left|\frac{\pi_{\Delta_{n}}(f \mid y)}{\pi_{\Delta_{n}}(f \mid \tilde{y})}-1\right|=\left|\prod_{j=1}^{N} \frac{\pi_{\Delta_{n}}\left(f \mid y^{(j-1)}\right)}{\pi_{\Delta_{n}}\left(f \mid y^{(j)}\right)}-1\right| \leqslant\left|\prod_{j=1}^{N} \frac{\nu_{\Delta_{n}}\left(\mathrm{~d} x_{\Lambda} \mid y^{(j-1)}\right)}{\nu_{\Delta_{n}}\left(\mathrm{~d} x_{\Lambda} \mid y^{(j)}\right)}-1\right| \leqslant 2 \sum_{\ell \in \partial_{\mathrm{L}} \Delta_{n}} \sum_{\ell \in \Lambda} S_{\Delta_{n}}\left(\ell, \ell^{\prime}\right)
$$


which by (3.1) yields that $\mu=\tilde{\mu}$.

Proof of Theorem 1.8. If $\varkappa(V) \leqslant \varkappa_{*}$ for a certain $\varkappa_{*}>0$, then by (2.3), it follows that

$$
S_{\Delta}\left(\ell, \ell^{\prime}\right) \leqslant \sum_{\vartheta\left(\ell, \ell^{\prime}\right)} \varkappa_{*}^{\left|\vartheta\left(\ell, \ell^{\prime}\right)\right|}
$$

where the summation is taken over all self-avoiding paths. We fix any $\Lambda \in \mathfrak{L}_{\text {fin }}$ and choose a strictly increasing sequence $\left\{p_{n}\right\}_{n \in \mathbb{N}} \subset \mathbb{N}$, such that $\Lambda$ is contained in every ball $B_{p_{1}}(b)=\{\ell \in \mathrm{L} \mid \rho(\ell, b) \leqslant$ $\left.p_{1}\right\}$ with $b \in \Lambda$. Then we fix any $b \in \Lambda$ and set $\Delta_{n}=B_{p_{n}}(b)$ and

$$
N_{n}=\inf _{\ell \in \Lambda, \ell^{\prime} \in \partial_{\mathrm{L}} \Delta_{n}} \rho\left(\ell, \ell^{\prime}\right) \text {. }
$$

Thereby, $N_{n} \rightarrow+\infty$, as $n \rightarrow+\infty$, and one finds $n_{*} \in \mathbb{N}$, such that $N_{n} \geqslant N_{\ell}$, for all $n \geqslant n_{*}$ and $\ell \in \Lambda$, where $N_{\ell}$ is the same as in Definition 1.2. Take $\varkappa_{*}<\eta^{-1}$, where $\eta$ is as in Definition 1.2. Then, for any $\ell \in \Lambda$, one has

$$
\sum_{\ell^{\prime} \in \partial_{\llcorner} \Delta_{n}} S_{\Delta_{n}}\left(\ell, \ell^{\prime}\right) \leqslant C \sum_{N=N_{n}}^{+\infty}\left(\varkappa_{*} \eta\right)^{N},
$$

which yields (3.1) and thereby completes the proof of the theorem.

Finally, let us show that the Bassalygo-Dobrushin graphs are sparse.

Lemma 3.2. Suppose that the distance $\rho$ has the property (1.3), (1.4). Then the graph is sparse in the sense of Definition 1.2.

Proof. By induction, one can show that

$$
\left|\mathcal{L}_{N}(b)\right| \leqslant n_{b} \max \left\{n_{\ell_{1}} \cdots n_{\ell_{N-1}} n_{\ell_{N}}\right\},
$$

where the maximum is taken over all self-avoiding paths $\left\{b, \ell_{1}, \ldots, \ell_{N-1}, \ell_{N}\right\}$. Thus, if $\hat{n}=\sup _{\ell} n_{\ell}$ is finite, then

$$
\left|\mathcal{L}_{N}(b)\right| \leqslant \hat{n} \cdot \hat{n}^{N}
$$

In case $\sup _{\ell} n_{\ell}=+\infty$, for given $p \in \mathbb{N}$, we let $B_{p}=\{\ell \in \mathrm{L} \mid \rho(\ell, b) \leqslant p\}$ and $n_{p}=\max _{\ell \in B_{p}} n_{\ell}$. Suppose that $n_{p}>n_{b}$, which can be achieved by taking big enough $p$. Then we pick $\ell_{p} \in B_{p}$, such that $n_{p}=n_{\ell_{p}}$, and $d_{p} \in \mathbb{N}$, such that

$$
\max \left\{p, \phi\left(n_{p}\right)\right\}-1 \leqslant 2 d_{p} \leqslant \max \left\{p, \phi\left(n_{p}\right)\right\} .
$$

Let us show that $n_{\ell} \leqslant n_{p}$ for every $\ell \in B_{d_{p}}$. If $p \geqslant \phi\left(n_{p}\right)$, then $B_{d_{p}} \subset B_{p}$ and $n_{\ell} \leqslant n_{p}$ by the definition of $n_{p}$. This is also true for $\phi\left(n_{p}\right) / 2 \leqslant p \leqslant \phi\left(n_{p}\right)$, as $B_{d_{p}}$ is still a subset of $B_{p}$. If $p \leqslant \phi\left(n_{p}\right) / 2$, then for any $\ell \in B_{d_{p}}$,

$$
\rho\left(\ell, \ell_{p}\right) \leqslant \rho(\ell, b)+\rho\left(b, \ell_{p}\right) \leqslant d_{p}+p \leqslant \phi\left(n_{p}\right) .
$$

Then $n_{\ell} \leqslant n_{p}$ by (1.3). Now let the path $\vartheta(b, \ell)$ be of length $N$ with $d_{p} / 2 \leqslant N \leqslant d_{p}$. Then it does not leave $B_{d_{p}}$ and hence $n_{\ell_{i}} \leqslant n_{p}$ for any $\ell_{i} \in \vartheta(b, \ell)$. Let $k_{p} \in \mathbb{N}$ be the largest possible $k$, for which $2^{k} \leqslant n_{p}$. Given $\ell_{i}, \ell_{j} \in \vartheta(b, \ell)$, such that $2^{k} \leqslant n_{\ell_{i}}, n_{\ell_{j}} \leqslant 2^{k+1}$, by $(1.3)$ one has $\rho\left(\ell_{i}, \ell_{j}\right) \geqslant \phi\left(2^{k}\right)$. Therefore, the number of such vertices in $\vartheta(b, \ell)$ does not exceed

$$
m_{k}=\frac{N}{\phi\left(2^{k}\right)}+1=\frac{N+\phi\left(2^{k}\right)}{\phi\left(2^{k}\right)} \leqslant \frac{5 N+1}{\phi\left(2^{k}\right)},
$$

where we have taken into account that $N \geqslant d_{p} / 2 \geqslant\left(\phi\left(n_{p}\right)-1\right) / 4$. Thereby, for chosen $N$, by (3.5) it follows that, see (1.4),

$$
\left|\mathcal{L}_{N}(b)\right| \leqslant \prod_{k=0}^{k_{p}} 2^{(k+1) m_{k}} \leqslant \exp \left\{\ln 2 \sum_{k=0}^{+\infty} \frac{k+1}{\phi\left(2^{k}\right)}(5 N+1)\right\} .
$$

Then the constants $C$ and $\eta$ can be calculated from the latter expression. To find $N_{b}$ one picks $p$ such that $n_{p}>n_{b}$. Then as $N_{b}$ one takes the smallest $N$ obeying $N \geqslant d_{p} / 2$. 


\section{A dense graph}

Here we consider an example of the dense graph mentioned in the introduction. The graph is an expanding tree; its set of vertices is

$$
\mathrm{L}=\bigcup_{n=0}^{+\infty} \mathrm{L}_{n} .
$$

The set $\mathrm{L}_{0}=\{b\}$ is a singleton and $\mathrm{L}_{1}=\partial_{\mathrm{L}} b, n_{b}=k$. Each $\ell \in \mathrm{L}_{n}, n \geqslant 1$, is adjacent to $k+n-1$ elements of $\mathrm{L}_{n+1}$ and with one element of $\mathrm{L}_{n-1}$. Here $k \geqslant 2$ is a fixed number; there are no connections within each of $\mathrm{L}_{n}$, see figure 1 .

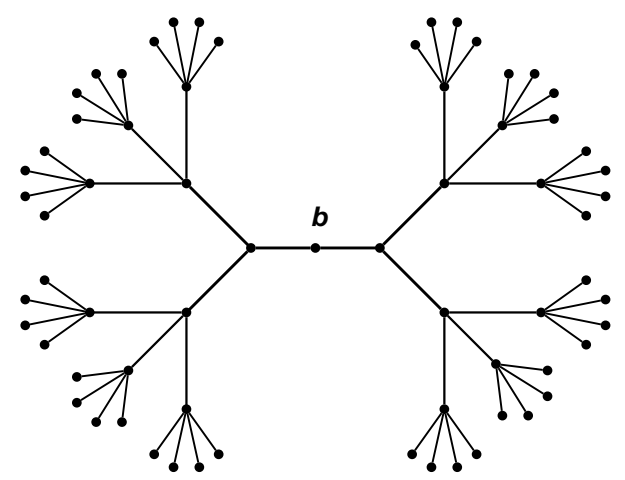

Figure 1. A dense graph for $k=2$.

For this graph, one has

$$
\left|\mathcal{L}_{N}(b)\right|=\frac{k(k+N-2) !}{(k-1) !} ;
$$

hence, it does not possess the property established by Definition 1.2.

On this graph, we define a ferromagnetic Ising model: for each $\ell \in \mathrm{L}$, we set $X_{\ell}=\{1,-1\}$ and $\chi_{\ell}(\{1\})=\chi_{\ell}(\{-1\})=1 / 2$. Furthermore, for $\left\langle\ell, \ell^{\prime}\right\rangle \in \mathrm{E}$, we set

$$
V_{\ell \ell^{\prime}}\left(x_{\ell}, x_{\ell^{\prime}}\right)=a x_{\ell} x_{\ell^{\prime}}, \quad a>0,
$$

and $\Delta_{n}=\cup_{s=0}^{n} \mathrm{~L}_{s}$. Therefore, $\partial_{\mathrm{L}} \Delta_{n}=\mathrm{L}_{n+1}$. Let $\nu_{\Delta_{n}}^{+}, n \in \mathbb{N}$, be the measure (1.18) corresponding to $y=\left(y_{\ell}\right)_{\ell \in \mathrm{L}}$ such that $y_{\ell}=+1$ for all $\ell \in \mathrm{L}$. Let also $\sigma_{n}$ be the projection of $\nu_{\Delta_{n}}^{+}$onto $\mathcal{B}\left(X_{b}\right)$. To simplify notations, we write $\sigma_{n}(\xi)=\sigma_{n}(\{\xi\}), \xi= \pm 1$. Our aim is to show that, for arbitrary $a>0$,

$$
\lim _{n \rightarrow+\infty} \frac{\sigma_{n}(1)}{\sigma_{n}(-1)}>1
$$

which means that the corresponding limiting Gibbs field ${ }^{1}$ is not invariant under the change of signs $x_{\ell} \mapsto-x_{\ell}$, for all $\ell \in \mathrm{L}$; hence, $\mathcal{G}$ is never singleton. To this end we construct the following recurrence. Given non-negative integer $s$, let us take some $\ell \in \mathrm{L}_{s}$ and then set $\Lambda_{s}=\{\ell\}$, and

$$
\Lambda_{s+1}=\partial_{\mathrm{L}} \Lambda_{s} \cap \mathrm{L}_{s+1}, \quad \Lambda_{s+2}=\partial_{\mathrm{L}} \Lambda_{s+1} \cap \mathrm{L}_{s+2}, \ldots, \Lambda_{n+1}=\partial_{\mathrm{L}} \Lambda_{n} \cap \mathrm{L}_{n+1}, \ldots, .
$$

For $n \geqslant s$, we let

$$
\Lambda \stackrel{\text { def }}{=} \bigcup_{q=s}^{n} \Lambda_{q} .
$$

By $\sigma_{n}^{s}, s=0,1, \ldots, n+1$, we denote the projection of the measure $\nu_{\Lambda}$ with the boundary condition $y_{\ell^{\prime}}=+1$ for all $\ell^{\prime} \in \Lambda_{n+1}$. Then $\sigma_{n}^{n+1}(\xi)=\delta_{\xi, 1}$ and $\sigma_{n}^{0}=\sigma_{n}$. Thereafter, the recurrence in

\footnotetext{
${ }^{1}$ Its existence can be proven by repeating the proof of Proposition 1.7.
} 
question is

$$
\sigma_{n}(\xi)=\frac{1}{Z_{n}} \prod_{j=1}^{k}\left(\sum_{\xi_{j}= \pm 1} e^{a \xi \xi_{j}} \sigma_{n}^{1}\left(\xi_{j}\right)\right)
$$

and for $s \geqslant 1$,

$$
\sigma_{n}^{s}(\xi)=\frac{1}{Z_{n, s}} \prod_{j=1}^{k+s-1}\left(\sum_{\xi_{j}= \pm 1} e^{a \xi \xi_{j}} \sigma_{n}^{s+1}\left(\xi_{j}\right)\right)=\frac{1}{Z_{n, s}}\left[e^{a \xi} \sigma_{n}^{s+1}(1)+e^{-a \xi} \sigma_{n}^{s+1}(-1)\right]^{k+s-1}
$$

where $Z_{n, s}$ is a normalizing factor. Now we set $u_{n, s}=\sigma_{n}^{s}(1) / \sigma_{n}^{s}(-1)$ and $u_{n}=u_{n, 0}$. Then by (4.5) it follows that

$$
u_{n}=\left(\frac{e^{2 a} u_{n, 1}+1}{u_{n, 1}+e^{2 a}}\right)^{k}, \quad u_{n, s}=\left(\frac{e^{2 a} u_{n, s+1}+1}{u_{n, s+1}+e^{2 a}}\right)^{k+s-1}, \quad u_{n, n}=e^{2 a(k+n-1)} .
$$

For $p \in \mathbb{N}$ and $t, \gamma \geqslant 1$, let us consider

$$
\begin{aligned}
\psi_{p}(t, \gamma) & \stackrel{\text { def }}{=}\left(\frac{\gamma t+1}{t+\gamma}\right)^{p}=\sum_{l=0}^{p} \frac{p !}{l !(p-l) !} \cdot\left(\frac{(t-1)(\gamma-1)}{t+\gamma}\right)^{l} \\
& \geqslant 1+p \cdot \frac{(t-1)(\gamma-1)}{t+\gamma}
\end{aligned}
$$

Since $u_{n, s}=\psi_{k+s-1}\left(u_{n, s+1}, e^{2 a}\right)$, we have

$$
\begin{aligned}
u_{n, s}-1 & \geqslant(k+s-1) \cdot \frac{\left(u_{n, s+1}-1\right)\left(e^{2 a}-1\right)}{u_{n, s+1}+e^{2 a}} \\
& \geqslant \cdots \geqslant\left(\frac{(k+p-2) !}{(k+s-2) !} \cdot \prod_{q=1}^{p-s} \frac{e^{2 a}-1}{u_{n, s+q}+e^{2 a}}\right) \cdot\left(u_{n, p}-1\right),
\end{aligned}
$$

which holds for any $p \geqslant s$. For any fixed $\gamma>1$ and for $p>(\gamma+1) /(\gamma-1)$, there exists $\varepsilon \in(0,1)$, such that $\psi_{p}\left(\gamma^{\varepsilon}, \gamma\right) \geqslant \gamma^{\varepsilon}$. As $u_{n, n}=e^{2 a(k+n-1)}$, one has,

$$
u_{n, p} \geqslant e^{2 \varepsilon a}, \quad \text { for all } n>p>\left(e^{2 a}+1\right) /\left(e^{2 a}-1\right) .
$$

Let $p$ be the smallest such number, which means that $u_{n, q}<e^{2 \varepsilon a}<e^{2 a}$ for all $q<p$. Then by (4.6) and (4.8), it follows that for all $n>p$,

$$
u_{n, 1}-1 \geqslant \frac{(k+p-2) !}{2^{p-1}(k-1) !} \cdot\left(1-e^{-2 a}\right)^{p-1} \cdot\left(e^{2 \varepsilon a}-1\right)>0
$$

which readily yields (4.3).

\section{Acknowledgement}

The authors are grateful to Ph. Blanchard and V. Ustymenko for valuable discussions. The financial support by the DFG through the project 436 POL 113/115/0-1 and through SFB 701 "Spektrale Strukturen und topologische Methoden in der Mathematik" is cordially acknowledged. 


\title{
References
}

1. Georgii H.-O. Gibbs Measures and Phase Transitions, Studies in Mathematics, 9, Walter de Gruyter, Berlin-New York, 1988.

2. Bassalygo L.A., Dobrushin R.L. Uniqueness of a Gibbs Field with a Random Potential - an Elementary Approach. Teor. Veroyatnost. i Primenen., 1986, 31, 651-670 (in Russian) [English translation: Theory Probab. Appl., 1987, 31, 572-589].

3. Albeverio S., Kondratiev Yu.G., Minlos R.A., Shchepan'uk G.V. Ground state Euclidean Gibbs measures for quantum lattice systems on compact manifolds, Rep. Math. Phys., 2000, 45, 419-429.

4. Albeverio S., Kondratiev Yu.G., Minlos R.A., Shchepan'uk G.V. Uniqueness problem for quantum lattice systems with compact spins, Lett. Math. Phys., 2000, 52, 185-195.

5. Kozitsky Yu., Pasurek T. Euclidean Gibbs measures of interacting quantum anharmonic oscillators. J. Stat. Phys., 2007, 127, 985-1047.

\section{Єдиність типу Бассалиго-Добрушин для систем 3 неперервним спіном на регулярних графах}

\author{
Д.Кепа, Ю.Козицький \\ Інститут математики, Університет Марії Кюрі-Склодовської, Люблін, Польща \\ Отримано 31 січня 2008 р.
}

Подається розширення техніки Бассалиго-Добрушина для доведення єдиності гіббсових полів на нерегулярних графах для систем неперервних спінів.

Ключові слова: випадкові гіббсові поля, специфікація Гіббса, Польський простір

PACS: $05.20 . \mathrm{Gg}, 05.30 . \mathrm{Ch}$ 
